\title{
Mechanisms of Cellular Avidity Regulation in CD2-CD58-Mediated T Cell Adhesion
}

\author{
De-Min Zhu ${ }^{\dagger, \neq}$, Michael L. Dustin ${ }^{\S}$, Christopher W. Cairo ${ }^{\dagger, \uparrow}$, Hemant S. Thatte ${ }^{\dagger, \|}$, and

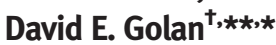 \\ ${ }^{\dagger}$ Departments of Biological Chemistry and Molecular Pharmacology, Surgery, and Medicine, Harvard Medical School, \\ Boston, Massachusetts 02115, §Skirball Institute of Biomolecular Medicine and the Department of Pathology, NYU School \\ of Medicine, New York, New York 10016, "Department of Surgery, Veterans Affairs Boston Healthcare System, West \\ Roxbury, Massachusetts 02132, and **Hematology Division, Brigham and Women's Hospital, Boston, Massachusetts \\ 02115, ${ }^{\ddagger}$ Current address: WP78-302, Merck Research Laboratories, Merck \& Co. Inc., West Point, Pennsylvania 19486, \\ "Current address: Department of Chemistry, University of Alberta, Edmonton, Alberta T6G 2G2, Canada
}

$\mathrm{T}$ he interaction of T lymphocytes with antigen presenting cells (APCs) and target cells is critically dependent on CD2-CD58-mediated adhesion (1, 2). Antibody blocking of CD2 prevents $T$ cell receptor (TCR)-mediated cell activation, and CD2 itself contributes to cell activation $(3,4)$. CD2 localizes to the T cell uropod, where the fast on and off rates of the receptor facilitate scanning of $\operatorname{APCs}(5,6)$. The low molecular profile of the CD2-CD58 complexes allows these molecules to integrate with the TCR-peptide-bound major histocompatibility complex (pMHC) complexes $(7,8)$, providing a permissive environment in which the lowaffinity TCR-pMHC interactions (9-13) can achieve the single receptor sensitivity required for antigen recognition (14). CD2-mediated T cell adhesion is modulated by the activation state of the cell. T cell activation, induced by TCR engagement or by phorbol-12-myristate-13acetate (PMA) stimulation, has been reported to cause a 2.5-fold enhancement of CD2-mediated T cell adhesion (15). Several molecular mechanisms have been proposed to regulate CD2-mediated adhesion, including changes in the conformation and cytoskeletal interaction of CD2 (6), although the biophysical manifestations of CD2 adhesion modulation are not well defined.

Based on the results of experiments performed in solution, the CD2-CD58 interaction has a low equilibrium binding affinity and a fast kinetic dissociation rate $(16-18)$. In the cellular context, however, adhesion receptors interact in a $2 \mathrm{D}$ rather than a $3 \mathrm{D}$ presentation; in the $2 \mathrm{D}$ environment, receptor mobility is restricted by association with the plasma membrane and by attachment to cytoskeletal proteins. At sites of cell-cell
AB STRACT The CD2 receptor on T lymphocytes is essential for T cell adhesion and stimulation by antigen presenting cells (APCs). Blockade of CD2 function is immunosuppressive in both model systems and humans, indicating the importance of CD2 for the cellular immune response. Although the affinity of the molecular interaction between CD2 and its counter-receptor, CD58, is relatively low when measured in solution, this interaction mediates tight adhesion within the 2D cellcell interface. To understand the mechanisms responsible for regulating the avidity of the CD2-CD58 interaction, we measured the number, affinity, and lateral mobility of CD2 molecules on resting and activated T cells. Cell activation caused a 1.5 -fold increase in the number of CD2 sites on the cell surface, and the 2D affinity of $\mathrm{CD} 2$ for $\mathrm{CD} 58$ increased by 2.5 -fold. The combination of T cell activation and CD2 ligation to CD58 decreased the laterally mobile fraction of the ligated CD2. Together, these changes would substantially enhance CD2 avidity and strengthen T cell-APC adhesion. The change in CD2 mobile fraction suggests that the cell uses cytoskeletal regulators to immobilize the receptor selectively at the site of contact with surfaces expressing CD58. Our observations are consistent with a model in which T cell activation initially induces increased CD2 2D affinity, cell surface receptor expression, and lateral mobility, allowing the CD2 molecules to diffuse to sites of contact with CD58-bearing APCs. Subsequently, T cell activation causes the CD58-bound CD2 to be recognized and immobilized at sites of cell-cell contact, thereby strengthening T cell-APC adhesion.

\section{*Corresponding author,} dgolan@hms.harvard.edu.

Received for review June 14, 2006 and accepted October 23, 2006. Published online November 17, 2006 10.1021/cb6002515 CCC: \$33.50 (c) 2006 by American Chemical Society 


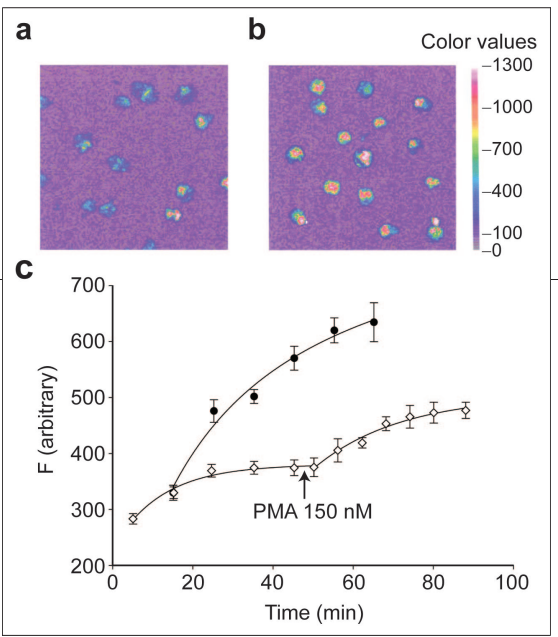

contact, dynamic polyvalent interactions between receptors and counter-receptors result from the mobile nature of receptors in biological membranes and the presentation of multiple binding sites on each cell, particularly as the systems approach equilibrium. Therefore, the physiological CD2-CD58 interaction can be appreciated only by determining the $2 \mathrm{D}$ affinity of the interacting partners. The 2D dissociation constant is defined by the law of mass action as $2 \mathrm{D} K_{\mathrm{d}}=[\mathrm{CD} 2] \times[\mathrm{CD} 58] /[\mathrm{CD} 2-\mathrm{CD} 58]$, where [CD2], [CD58], and [CD2-CD58] represent surface densities of free $C D 2$, free $C D 58$, and bound CD2-CD58 complexes in molecules $\mu \mathrm{m}^{-2}$, with the $2 \mathrm{D} K_{\mathrm{d}}$ in the same units $(19,20)$.

It has been difficult to obtain experimental data of suitable quality for $2 \mathrm{D} K_{\mathrm{d}}$ measurements, both because of the technical difficulties of observing receptors in an adhesion between two cells and because of the problem of deconvolving adhesion receptor-counterreceptor interactions in the interface from cytoskeletal and membrane domain interactions with the receptors. A breakthrough was achieved in both respects by the application of supported planar bilayers (21). Here, glycosylphosphatidylinisotol (GPI)-linked CD58 is fluorescently labeled and reconstituted into planar phospholipid bilayers to simulate the membrane of an APC, and $T$ cell adhesion to CD58 is observed by accumulation of CD58 fluorescence at the cell-bilayer interface (22). This experimental method allows measurement of free bilayer CD58 density (molecules $\mu \mathrm{m}^{-2}$ ), cellbilayer contact area $\left(\mu \mathrm{m}^{2}\right)$, and the density of CD2CD58 interactions in the contact area (molecules $\mu \mathrm{m}^{-2}$ ) at equilibrium. We have also incorporated a linearized plot for calculation of the $2 \mathrm{D} K_{\mathrm{d}}$ of the CD2-CD58 interaction and the total number of laterally mobile CD2 molecules on the cell surface (23). We refer to affinity as the inverse of the $2 \mathrm{D} K_{\mathrm{d}}$ measured by the Zhu-Golan analysis $(22,24)$, and we refer to avidity as the total adhesive strength of the interaction. Two previous studies utilized this experimental and analytical approach and found that the CD2-CD58 interaction should result in strong adhesion, with a $2 \mathrm{D} K_{\mathrm{d}}$ of 1.1-7.6 molecules $\mu \mathrm{m}^{-2}$ for resting Jurkat $\mathrm{T}$ cells adhering to model bilayers reconstituted with laterally mobile CD58 at $24^{\circ} \mathrm{C}(23$, 25). A comparison of this $2 \mathrm{D} K_{\mathrm{d}}$ with the physiological densities of CD2 and CD58 on T cell and APC surfaces, respectively, suggests that $>85 \%$ of $C D 2$ molecules are bound to CD58 in the contact area.

Here we apply our T cell-planar bilayer system to identify and characterize the mechanisms that regulate the avidity of T cell CD2 for its counter-receptor CD58. We quantify the number of receptors, receptor affinity, and receptor lateral mobility on both resting and activated T cells. By using quantitative fluorescence imaging, fluorescence photobleaching recovery (FPR), and single particle tracking (SPT), we observe that cell activation causes an increased rate of CD58 accumulation and an increased density of CD58 at the contact area. This increased accumulation is due to increases in cell surface CD2 expression and receptor affinity. We also provide evidence that CD2 is selectively immobilized at the contact site, further enhancing the number of potential CD2-CD58 bonds in the adhesion zone.

\section{RESULTS AND DISCUSSION}

Accumulation of CD58 at the Contact Area. Jurkat T cells were used as a model, because CD2 expressed on these cells has a 2D affinity for CD58 similar to that of human peripheral blood lymphocytes (23). Jurkat cells adhered to bilayers containing FITC-CD58 and caused the local density of CD58 to increase at the contact areas. This interaction was specifically inhibited by the adhesion-blocking monoclonal antibody (mAb) TS2/18 (24), and it was consistent with the mass-action equation for CD2 binding to CD58. We tested the effect of cell activation on CD58 accumulation by comparing the accumulation of fluorescence in the contact area for control cells with that for cells stimulated with PMA. After equivalent incubation times, the accumulated fluorescence in the contact area was greater for PMAstimulated cells than for control cells (Figure 1, panels a and b). Control cells showed a plateau of accumulated CD58 after 30-40 min of incubation (Figure 1, panel c), 
consistent with previous results $(22,25)$. Introduction of PMA after this time period caused an additional increase in CD58 accumulation. Similarly, cells stimulated with PMA for 20 min before incubation with CD58-containing bilayers showed a higher density of accumulated CD58 at equilibrium and required longer for the density to reach a plateau. These experiments indicated that the cell surface expression, binding affinity, or lateral mobility of CD2 molecules or a combination of these was altered on PMA-stimulated cells. The rate of CD58 accumulation was also 2 -fold faster in PMA-treated cells than in control cells, probably due to the higher CD2 surface density on the activated cells (vide infra).

Consistent with the mass-action equation, the initial density of CD58 in the bilayer was an important determinant of the level of CD58 accumulation at equilibrium. We determined the plateau level of CD58 accumulation as a function of initial CD58 density for control and PMAtreated cells (Figure 2, panel a). PMA stimulation caused the equilibrium density of CD58 in the contact area to increase. The maximum density of CD 58 was $\sim 650$ molecules $\mu \mathrm{m}^{-2}$ for control cells and $\sim 950$ molecules $\mu \mathrm{m}^{-2}$ for PMA-activated cells. Cell stimulation did not produce a significant change in the contact area of the cells: the maximum contact area size was $65 \mu \mathrm{m}^{2}$ per cell for resting cells and $68 \mu \mathrm{m}^{2}$ per cell for PMAactivated cells. From these data, we confirmed that CD58 accumulation (signifying the accumulation of CD2-CD58 complexes) depended directly on the initial CD58 density and determined that CD58 accumulation also depended on the activation state of the cell.

Lateral Mobility of CD58 in the Bilayer. Accumulation of $C D 58$ in the contact area could have been related to a change in the lateral mobility of CD58 in the bilayer as a result of binding to $C D 2$. We used FPR to measure the mobility of CD58 within and outside the cell-bilayer contact area (Table 1). FPR was performed under conditions that did not deplete FITC-CD58 in the contact area, such that diffusion of CD58 from the bilayer into the contact area was not a limiting factor. The diffusion coefficient $(D)$ of FITC-CD58 in bilayer regions outside the contact area was high, as expected for diffusion of a GPIlinked protein in a model bilayer membrane. Within the area of contact with resting cells, the diffusion coefficient of CD58 was reduced. Treatment of cells with PMA further slowed CD58 diffusion in the contact area. The fractional mobility $(A)$ of FITC-CD58 showed a similar trend: virtually no immobile CD58 was observed outside the contact area $(f=94 \%$ ), while progressive (albeit modest) CD58 immobilization was found within the area of contact with resting and PMA-activated cells. Therefore, dynamic interaction with CD2 on the cell surface caused reduced mobility of CD58 in the contact area, as previously observed (22).

Lateral Mobility of CD2 on Cells. CD2 was labeled with the FITC-conjugated non-adhesion-blocking mAb CD2.1 to determine the lateral mobility of CD2 on cells adherent to bilayers reconstituted with unlabeled CD58. A low initial CD58 density was used to minimize migration of CD2 to the contact area while allowing cells to adhere to the bilayer. Measurements of CD2 lateral mobility were performed at the surface of the cell in contact with the bilayer and at the opposite surface of the cell not in contact with the bilayer (Table 1). The diffusion coefficient of CD2 was $(6-7) \times 10^{-10} \mathrm{~cm}^{2} \mathrm{~s}^{-1}$ in both resting and PMA-treated cells. This observation was consistent with previous results (26) and with the diffusion coefficient of other adhesion receptors on Jurkat cells, e.g., the lateral diffusion coefficient of LFA-1 is $(5-7) \times 10^{-10} \mathrm{~cm}^{2} \mathrm{~s}^{-1}$, depending on the mAb used to label the receptor $(27,28)$.

The fractional mobility of CD2 was high outside the contact area, in resting and PMA-treated cells. In resting cells, CD2 fractional mobility was also high within the contact area, within experimental error of that outside the contact area (Table 1). However, in PMA-treated cells, the fractional mobility of CD2 within the contact area showed a dramatic and time-dependent decrease (Table 1). To characterize the time dependence of this immobilization, we measured CD2 fractional mobility in the area of contact with activated cells as a function of time (Figure 2, panel b). The fractional mobility of CD2 was low for the first $\mathbf{4 0}$ min after cell activation. The $f$ value then increased gradually to $50 \% 120 \mathrm{~min}$ after PMA treatment. These results suggested that i) the cell recognized the ligation of $C D 2$ by $C D 58$, ii) the cell selectively immobilized the bound CD2 only when the cell was activated, and iii) the cell gradually released the immobilized CD2 over a time period of several hours. Furthermore, the combination of profound CD2 immobilization and modest CD58 immobilization was consistent with the rapid off rate for CD2-CD58 binding (1618), and the slowing of CD58 diffusion in the context of cell activation was consistent with a reduced off rate (increased affinity) for CD2-CD58 binding. The selective immobilization of CD58-bound CD2 was likely due to 


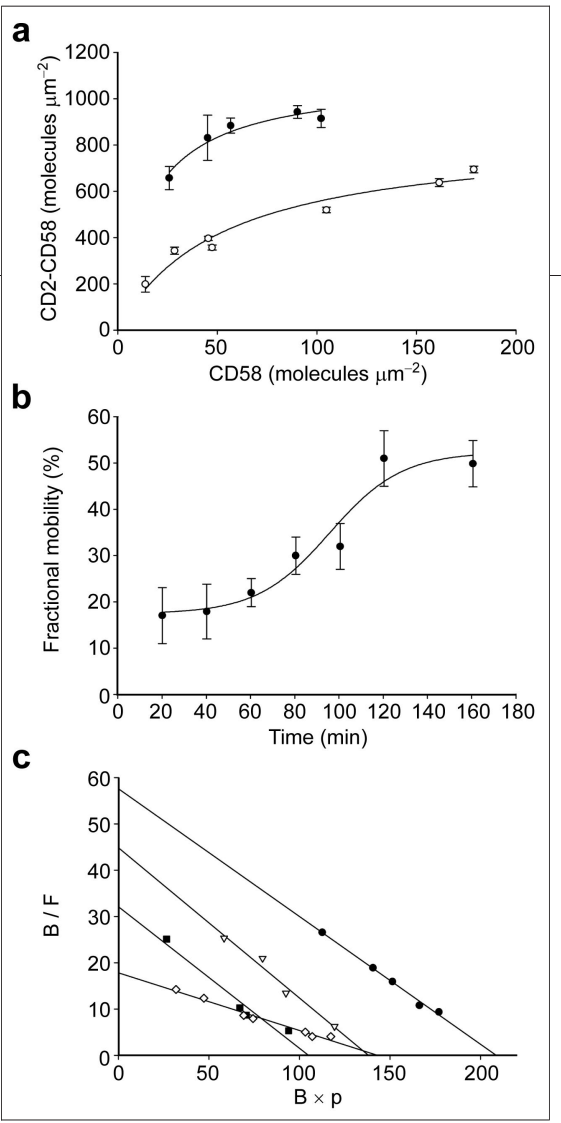

Figure 2. Effect of PMA activation on CD58 accumulation, CD2 lateral mobility, and CD2-CD58 affinity. a) Accumulation of bound CD58 molecules in the contact area as a function of CD58 density in the bilayer. Cells were incubated for $50 \mathrm{~min}$ with bilayers containing varying initial densities of CD58, and the density of bound CD58 (reflecting the density of CD2-CD58 complexes) was measured as described in the text: $(O)$ control cells; $(\Theta)$ cells pre-incubated with $150 \mathrm{nM}$ PMA for $20 \mathrm{~min}$.

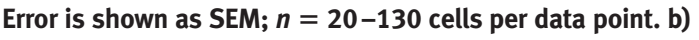
Time dependence of recovery from CD2 immobilization induced by the combination of CD2-CD58 binding and cell activation. Jurkat cells were incubated with bilayers containing 100-270 molecules $\mu \mathrm{m}^{-2} \mathrm{CD} 58$, and $150 \mathrm{nM}$ PMA was added immediately thereafter (time zero). CD2 was labeled with mAb FITCCD2.1, and FPR was used to determine the fractional mobility $(f)$ of CD2 in the contact area. Data points represent the mean values of $f$ for $9-28$ cells from 2-3 experiments at each point. Error is shown as SEM. c) Determination of 2D affinity (2D $K_{d}$ ) of CD2-CD58 interaction. The Zhu-Golan plot $(B / F$ vs $B \times p)$ was used to determine the $2 \mathrm{D}$ affinity of adhesion in control and activated Jurkat cells (23) (summarized in Table 2). Data are shown for the following: $(\diamond)$ control cells; $(\diamond)$ PMA-activated cells; $(\nabla)$ OKT3-GaM-treated cells; $(\square)$ CD2.1-treated cells.

protein kinase $\mathrm{C}(\mathrm{PKC})$; $\mathrm{CD} 3$ crosslinking with $\mathrm{mAb}$ OKT3 and secondary goat anti-mouse (GaM) antibody stimulates the cell through the TCR (18); and the pair of anti-CD2 mAbs FITC-TS2/18 and CD2.1 activates the cell through CD2. In these experiments, cells were examined on bare glass coverslips, not on CD58-containing bilayers. We observed that neither PMA nor CD2.1 altered the fractional mobility of FITC-TS2/18labeled CD2. Stimulation of cells through the TCR did

increased association of $\mathrm{CD} 2$ with the T cell cytoskeleton, and the slow release of immobilized CD2 was likely caused by time-dependent changes in the strength of the CD2- cytoskeleton association (vide infra). The time course of immobilized CD2 release could be consistent with that of cellular deadhesion required for rapid scanning of APCs by migrating T cells (29).

We also tested whether different modes of cell stimulation could affect CD2 lateral mobility in the absence of ligation by CD58 (Table 2). PMA activates the cell through result in a significant reduction in fractional mobility.

\section{Number and 2D Affinity of CD2 Molecules on Cells.}

FPR and quantitative fluorescence imaging experiments showed that cell activation alters the fractional mobility and density of CD2 in the contact area. We reasoned that the number of cell surface CD2 molecules and the $2 \mathrm{D}$ affinity of the CD2-CD58 interaction could also have a role in avidity regulation. We used the Zhu-Golan analysis (described in Methods and elsewhere $(23,24)$ ) to measure the number and affinity of $C D 2$ receptors in the

\section{TABLE 1. Lateral mobility of CD58 and CD2}

\begin{tabular}{|c|c|c|c|c|c|}
\hline FITC-labeled molecule & Treatment & Region & $f(\%)^{a}$ & $D\left[\times 10^{-10} \mathrm{~cm}^{2} \mathrm{~s}^{-1}\right]^{b}$ & $N$ \\
\hline FITC-CD $58^{c}$ & Control & Outside contact area & $94 \pm 2$ & $26.8 \pm 3.7$ & 20 \\
\hline FITC-CD58 ${ }^{c}$ & Control & Contact area & $87 \pm 2$ & $9.8 \pm 0.8$ & 32 \\
\hline FITC-CD58 ${ }^{c}$ & PMA-activated & Contact area & $81 \pm 2$ & $5.1 \pm 0.5$ & 53 \\
\hline FITC-CD2.1 $\mathrm{Ab}^{d}$ & Control & Top of celle & $77 \pm 6$ & $5.9 \pm 0.7$ & 18 \\
\hline FITC-CD2.1 $\mathrm{Ab}^{d}$ & Control & Contact area & $72 \pm 4$ & $6.9 \pm 0.7$ & 35 \\
\hline FITC-CD2.1 $\mathrm{Ab}^{d}$ & PMA-activated & Top of celle & $74 \pm 4$ & $7.3 \pm 0.9$ & 25 \\
\hline FITC-CD2.1 $\mathrm{Ab}^{d}$ & PMA-activated & Contact area & $15-50$ & $6.9 \pm 0.6$ & 12 \\
\hline
\end{tabular}

${ }^{a}$ Fractional mobility of the FITC-labeled molecule in the indicated region. Values represent mean $\pm \mathrm{SEM}$. ${ }^{b}$ Diffusion coefficient of the FITC-labeled molecule in the indicated region. For comparison, the diffusion coefficient of NBD-labeled phosphatidylethanolamine in egg PC planar bilayers was $80 \times 10^{-10} \mathrm{~cm}^{2} \mathrm{~s}^{-1}$. Values represent mean $\pm \mathrm{SEM}$. ${ }^{C}$ The initial density of FITC-CD58 in the glass-supported planar lipid bilayer was 200 molecules $\mu \mathrm{m}^{-2}$. ${ }^{d}$ The initial density of CD58 in the bilayer was 30 molecules $\mu \mathrm{m}^{-2}$ for measurements at top of cell and $100-270$ molecules $\mu \mathrm{m}^{-2}$ for measurements at contact area. Cell surface CD2 was labeled with FITC-CD2.1. ${ }^{e}$ Focal plane was $10 \mu \mathrm{m}$ above the plane of cell-bilayer contact. contact area. For a

range of initial CD58 densities in the glass-supported planar bilayer, plots of $B / F v s B \times p$ (see eq 2) were constructed from experimental measurements of bound CD2-CD58 complex density $(B)$, free CD58 density $(F)$, CD2 fractional mobility (f), and ratio of contact area to cell surface area (p). Cell surface area was calculated as 
$S_{\text {cell }}=4 \pi r^{2} \times 1.8$, where $r$ was the measured radius of the cell and 1.8 was a correction factor for surface roughness (30). We confirmed the validity of the $S_{\text {cell }}$ equation by osmotic swelling experiments (25). Jurkat cells had a mean cell

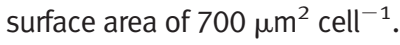

In the Zhu-Golan plots (Figure 2, panel c), the $X$-intercept was used to determine the number of CD2 receptors $\left(N_{\mathrm{t}}\right)$ according to eq 3 . The number of $C D 2$ receptors per cell was also determined using radiolabeled $\mathrm{mAb}$ binding (Table 2 ).

Because the mAb-binding method averages over all cells in a sample, whereas the Zhu-Golan method examines only cells that bind to CD58containing bilayers, the latter method is expected to be biased toward cells expressing high levels of CD2. Nonetheless, the measurements obtained using the two methods were within 1.5-2.5-fold of one another; as expected, estimates of $N_{\mathrm{t}}$ obtained using the mAbbinding method were systematically lower than those determined by the Zhu-Golan method. $N_{\mathrm{t}}$ values measured using the Zhu-Golan method showed that resting and CD2.1-labeled cells expressed similar numbers of CD2 molecules on the cell surface, whereas cells activated with PMA or OKT3-GaM showed a 1.6-fold increase in CD2 surface expression.

In a Zhu-Golan plot, the negative reciprocal of the slope provides the $2 \mathrm{D}$ affinity $\left(2 \mathrm{D} K_{\mathrm{d}}\right)$ of the $\mathrm{CD} 2-\mathrm{CD} 58$ interaction $(23,25)$. All three cell activation treatments resulted in a 2.5 -fold decrease in the $2 \mathrm{D} K_{\mathrm{d}}$ (Table 2 ). Taken together, we found that cell activation by PMA or OKT3-GaM induced an increase in CD2 expression and that cell activation by PMA, OKT3-GaM, or CD2.1CD58 caused an increase in receptor affinity. At equilibrium, the change in affinity would result in an increase from $87 \%$ to $96 \%$ of bound CD2 in the T cell-APC contact area. We concluded that increases in both $2 \mathrm{D}$ affinity and receptor expression were used by the cell to increase the avidity of the CD2-CD58 interaction. The magnitude and direction of this shift in CD2 avidity upon cell activation have been observed previously (15).

Our results are consistent with the involvement of a conformational change in CD2 as the basis for the increase in CD2 affinity, because treatment of T cells with the activating anti-CD2 mAb pair TS2/18 and CD2.1 produces an increase in affinity identical to that associated with cell activation through the PKC or TCR pathways $(15,31)$. Conformational changes in CD2 have

\begin{tabular}{|c|c|c|c|c|c|}
\hline \multirow[b]{2}{*}{ Treatment } & \multirow[b]{2}{*}{$f(\%)^{a}$} & \multicolumn{2}{|c|}{$N_{\mathrm{t}}\left[\times 10^{4}\right.$ molecules cell $\left.{ }^{-1}\right]$} & \multirow[b]{2}{*}{$2 \mathrm{D} K_{\mathrm{d}}{ }^{c}$} & \multirow[b]{2}{*}{$r^{2}$} \\
\hline & & mAb assay & $\begin{array}{l}\text { Zhu-Golan } \\
\text { analysis }\end{array}$ & & \\
\hline Control & $73 \pm 3$ & $4.3-8.6$ & 13.2 & 8.1 & 0.97 \\
\hline PMA & $70 \pm 4$ & $4.0-8.0$ & 20.9 & 3.6 & 0.99 \\
\hline OKT3-GaM & $35 \pm 2$ & $b$ & 21.0 & 3.3 & 0.96 \\
\hline CD2.1 & $74 \pm 4$ & $b$ & 13.1 & 3.1 & 0.97 \\
\hline
\end{tabular}

${ }^{a}$ Mean \pm SEM from $>40$ cells determined in $>3$ experiments. CD2 was labeled with FITCTS2/18. ${ }^{b}$ Not determined. ${ }^{c}$ Measured in molecules $\mu \mathrm{m}^{-2}$.

been observed by antibody labeling, immunofluorescence, and NMR studies (32-36). Other potential mechanisms for altered affinity, such as changes in the topology or intermembrane separation of the T cellbilayer interface, are unlikely to be important here because the interface is flat, uniform, and stable when the bilayer is reconstituted with CD58 (36).

Changes in CD2-CD58 affinity are likely to have a fundamental role in adhesion strengthening. A low $2 \mathrm{D} K_{\mathrm{d}}$ results in a small confinement region of the adhesion receptors $(19,25)$. Our findings indicate that the resting $T$ cell holds CD2 in a lower affinity conformation that is capable of engaging in an initial "loose" interaction with APCs and that cell stimulation (e.g., by TCR engagement) converts CD2 from the lower affinity conformation to a higher affinity form that mediates tighter adhesion through confinement of both receptors. We note that our initial report of CD2-CD58 affinity used a different clone of Jurkat cells than that used here (25); our current data (23, and the present study) suggest that the earlier clone could have been activated. Cell-based functional studies have found that PMA activation of a T cell hybridoma enhances by 2.5 -fold the avidity of adhesion mediated by the CD2-CD58 interaction (15).

The activation-induced increase in the number of CD2 molecules at the cell surface occurs within $1 \mathrm{~h}$ of cell activation, and it is therefore unlikely that synthesis of new CD2 molecules is responsible for the increase (37). Thatte et al. (38) (and others) have shown that transmembrane receptors are stored in cytoplasmic vesicles and that disruption of the microtubule network with colchicine can induce complete surface expression of the stored protein within $60 \mathrm{~min}$. Treatment of Jurkat cells with $10 \mu \mathrm{M}$ colchicine for 30 min causes a $60 \%$ 


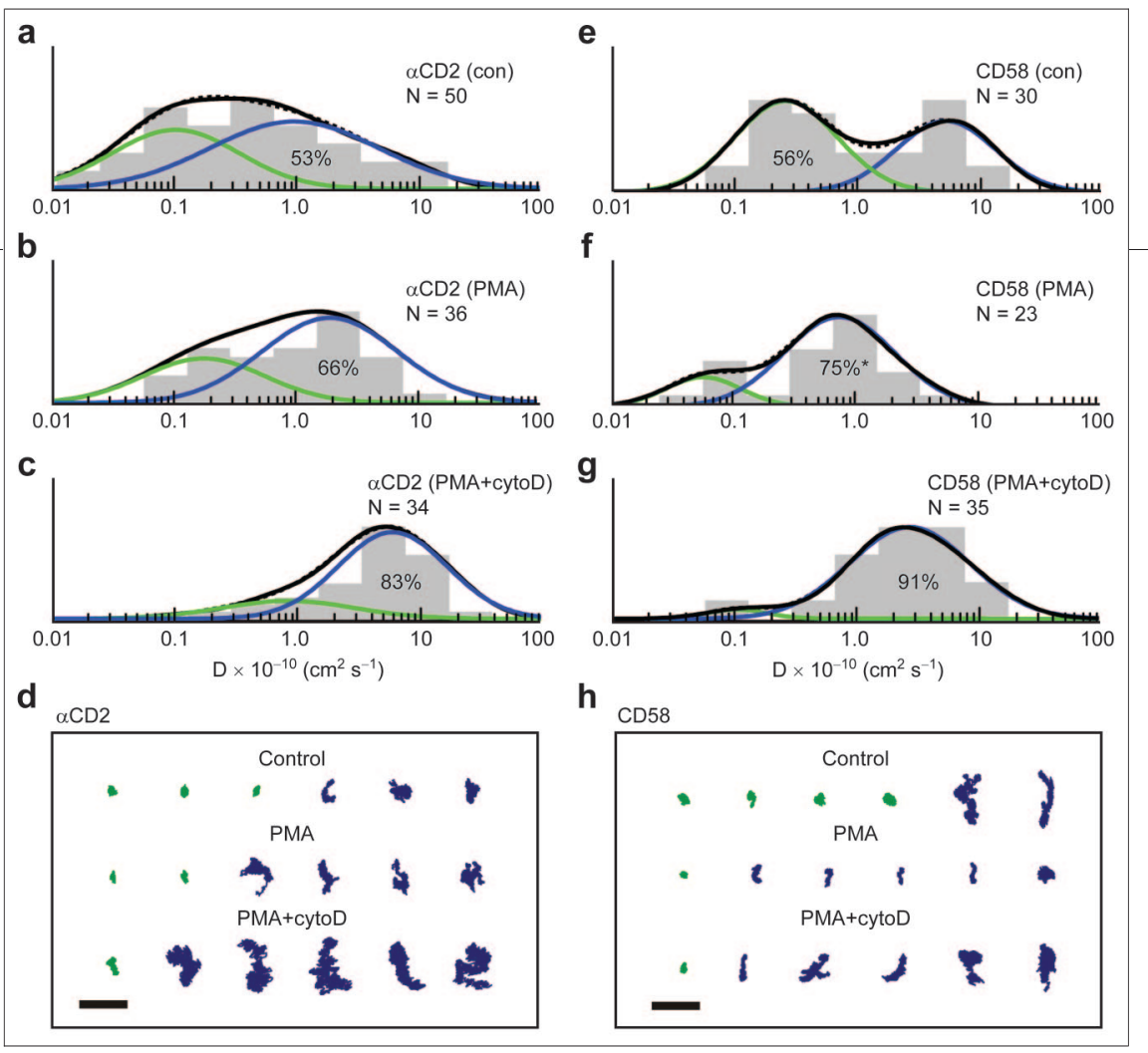

Figure 3. SPT of CD2. The diffusion of CD2 on Jurkat cells was observed by high-speed SPT. Beads were labeled with either $\mathrm{mAb}$ TS2/18 $F(a b)$ ' (a-d) or purified GPI-CD58 (e- h). Cells were treated for $30 \mathrm{~min}$ with buffer containing the following: a, e) DMSO (0.1\%); b, f) PMA (150 nM, 0.1\% DMSO); or c, g) cytoD and PMA (5 $\mu \mathrm{M}$ and $150 \mathrm{nM}, 0.1 \%$ DMSO). Tracking data were analyzed to determine the lateral diffusion coefficients corresponding to the trajectories of individual particles; these data are shown as a histogram (gray) and as a smoothed population density function (black line). The best fit of the population density is a dotted line, and the best-fitted subpopulations are green and blue lines. The relative fraction of the dominant subpopulation is indicated. Representative trajectories (2 s, 1000 FPS) are shown for control, PMA-treated, and cytoD+PMA-treated cells (d, h). * denotes a subpopulation showing directed motion. Scale bar $=1 \mu \mathrm{m}$.

increase in the surface expression of CD2 as measured by FITC-TS2/18 labeling (data not shown). Longer incubations with colchicine do not increase the intensity of FITC-TS2/18 fluorescence at the cell surface, indicating that the $C D 2$ reservoir is depleted with 30 min of incubation. These results suggest that $T$ cells retain a cytoplasmic pool of CD2 that can be rapidly translocated to the cell surface in order to increase CD2-mediated adhesion.

SPT of CD2 on Cells. Our FPR experiments suggested that the average lateral mobility of CD2 was regulated by cell activation and by ligand engagement. We employed SPT to provide a non-ensemble measurement of CD2 mobility on Jurkat cells (28). Measurements of lateral mobility using FPR are proportional to the average mobility of the population over relatively long times and distances and are therefore biased toward faster diffusion. In contrast, SPT measurements sample the population of molecules over shorter times and distances and are therefore more sensitive than FPR to slow diffusion. In addition, SPT is capable of resolving the presence of multiple subpopulations of molecules (27). Because SPT resolves the immobile population observed in FPR experiments, SPT and FPR often measure different mean values for lateral diffusion (27). Nonetheless, the two methods are complementary because they provide information about the properties of molecules on different time and distance scales (28). All SPT experiments labeled CD2 by using $1 \mu \mathrm{m}$ beads conjugated to either mAb TS2/18 or purified GPI-CD58, and all observations were made at the top surface of Jurkat cells mounted on glass coverslips. Complete SPT results are given in the supporting information (See Supplementary Tables 1-3).

The average lateral diffusion of CD2 observed by SPT was $\sim 3$-fold slower than that measured by FPR (Table 3), consistent with measurements on other cell surface proteins (28). Beads labeled with TS2/18 showed a small increase in average diffusion upon cell activation. Subpopulation analysis showed that there were two populations of trajectories within these data sets (Figure 3). Consistent with the FPR results, there was a significant slowly diffusing subpopulation in both resting and activated cells. The slow population was reduced upon cell activation; this change could have been responsible for the small increase in diffusion coefficient observed by FPR upon PMA stimulation of cells (Table 1). Because FPR measurements of lateral diffusion are biased toward faster diffusing receptors, we interpret the population of SPT trajectories centered at diffusion coefficients greater than $\sim(0.5-1.0) \times 10^{-10} \mathrm{~cm}^{2} \mathrm{~s}^{-1}$ to approximate the mobile fraction measured by FPR. The fractions of fasterdiffusing molecules in control and PMA-treated cells by SPT were reasonably consistent with FPR measurements of CD2 fractional mobility under the same conditions.

(See ref 39 for a more extended discussion of the differences between measurements of lymphocyte receptor lateral mobility by FPR and SPT.)

The bead used to label CD2 in SPT experiments was also employed to mimic a small area of CD2-CD58 
contact. Beads were conjugated to purified CD58 with a stoichiometry of $\sim 5000$ sites $\mu \mathrm{m}^{-2}$ and then used in SPT measurements on Jurkat cells. On resting cells, CD58-ligated CD2 showed a modest increase in average lateral diffusion relative to antibody-labeled CD2. Upon treatment with PMA, however, CD58-ligated CD2 showed significantly reduced lateral diffusion. Subpopulation analysis demonstrated that CD58-ligated CD2 was found in two major populations on resting cells. Cell activation changed the diffusion profile of CD58-ligated CD2: a small immobile population remained, but the major population showed an intermediate diffusion coefficient and evidence of directed motion (Figure 3). This analysis by SPT confirmed the FPR observation that CD2 mobility was synergistically affected by the combination of CD2-CD58 ligation and cell activation.

We also tested whether the effect of cell activation on CD2 lateral mobility was mediated by cytoskeletal interactions. Treatment of Jurkat cells with PMA and the actin cytoskeleton disrupting cytochalasin D (cytoD) revealed that the immobile populations of both antibody-labeled and CD58-ligated CD2 were constrained by interactions with the cytoskeleton (Figure 3). In both cases, cytoD treatment shifted the CD2 molecules into a single rapidly diffusing population. CytoD also increased the diffusion coefficients of CD2 in both cases (Table 3). The sensitivity of CD2 mobility to treatment with cytoD suggested that these molecules are attached directly or indirectly to the actin cytoskeleton. It is likely that this interaction is mediated through binding of the cytoplasmic tail of $C D 2$ to the $C D 2$ associated protein (CD2AP). CD2AP interacts dynamically with the proline-rich CD2 cytoplasmic tail (6), and it also contains a high-affinity binding site for the actin capping protein capZ (40).
Model for Cellular Regulation of CD2 Avidity. The avidity of cell adhesion depends on a number of different variables, and new methods are needed to dissect quantitatively the mechanisms that contribute to adhesion strength (avidity). We have employed a model adhesion system and a new receptor-ligand binding analysis to determine the $2 \mathrm{D}$ affinity of the CD2-CD58 interaction in a physiological environment. Our analysis takes account of the dynamic nature of the adhesion by incorporating experimental measurements of receptor lateral mobility $(22,24)$. We observe the effects of cell activation on receptor mobility using FPR and SPT. The two methods find similar results: CD2 mobility is only slightly affected by activation of the cell or by ligation to CD58, but there is a synergistic effect on lateral mobility when CD2 on activated cells is bound to CD58. We also find that cell activation increases the affinity of CD2 for CD58 by 2.5 -fold and the number of $\mathrm{CD} 2$ receptors at the cell surface by 1.5 -fold. Increased affinity, increased receptor density, and decreased lateral mobility at the contact area combine to make CD2CD58-mediated adhesion stronger and more selective (Figure 4). Our results provide the most complete analysis of CD2-mediated adhesion to date and demonstrate that CD2-CD58 avidity is regulated by the conjunction of cell activation and contact area; receptor conformational change and affinity; and receptor and ligand lateral mobility, expression level, and surface density.

\section{METHODS}

Cell Culture and Reagents. The Jurkat E6.1 T leukemia cell line (American Type Culture Collection) was maintained in RPMI
1640 medium (Sigma) supplemented with 10\% heat-inactivated fetal bovine serum (Sigma) and $2 \mathrm{mM}$ L-glutamine/100 units $\mathrm{mL}^{-1}$ penicillin/0.1 $\mathrm{mg} \mathrm{mL}^{-1}$ streptomycin (Sigma) at $37^{\circ} \mathrm{C}$ in a 


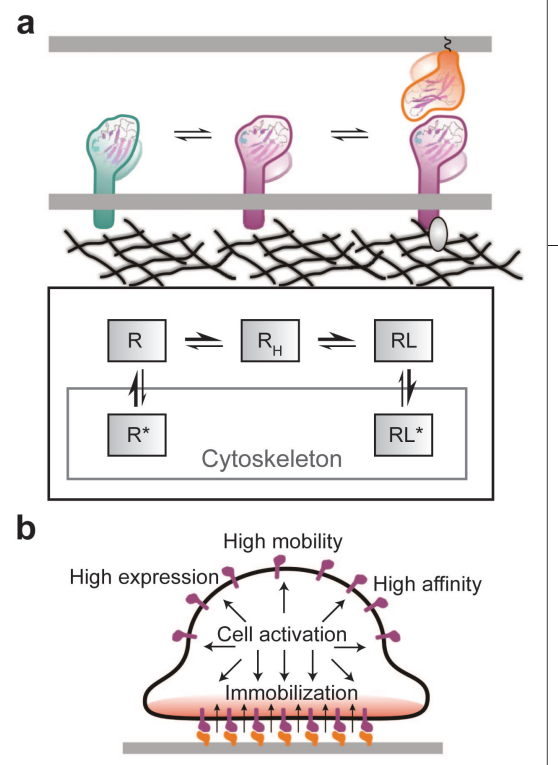

Figure 4. Model of CD2-CD58 avidity regulation. We propose a comprehensive model of CD2-CD58 avidity regulation that incorporates receptor affinity (2D $\left.K_{\mathrm{d}}\right)$, number $\left(N_{\mathrm{t}}\right)$, and lateral mobility $(f)$. a) CD2 $(R)$ is primarily mobile $(f \approx 50-70 \%)$ on resting cells, but there is a significant population of molecules that interacts with the cytoskeleton $\left(R^{*}\right)$. Activation of the cell by PMA or by TCR/CD3 cross-linking induces a conformational change that increases the affinity of CD2 for CD58 by $\sim 2.5$-fold $\left(R_{H}\right)$. The mobile, high-affinity CD2 molecules can diffuse laterally on the cell surface and engage CD58 within a contact area (RL). The bound receptor is preferentially immobilized on activated cells to promote adhesion (RL*), likely through association with CD2AP-capZ-actin complexes. b) Cellular activation results in increased expression, affinity, and lateral mobility of unligated CD2. At the contact area, CD2-CD58 ligation generates a second signal, likely through a second conformational change in CD2, that immobilizes the receptor selectively in the contact area. High lateral mobility allows receptors outside the contact area to diffuse into the contact area and engage CD58, thereby increasing the number of bound CD2-CD58 complexes and promoting adhesion. This model couples passive diffusion with active recognition of conformational change to strengthen adhesion.

humidified atmosphere of $5 \% \mathrm{CO}_{2}$. CytoD and PMA were purchased from EMD Biosciences. Other reagents were purchased from Sigma-Aldrich; cell media and buffers were purchased from Invitrogen. For SPT experiments, mAb TS2/18 was obtained from Endogen and digested to $F(a b)^{\prime}$ fragments by standard procedures. GPI-linked CD58 (GPI-CD58) was purified from human red cells as previously described (25).

Antibody Binding Assays. Jurkat cells were washed twice with $N$-(2-hydroxyethyl)piperazine- $N^{\prime}$-ethanesulfonic acid (HEPES)buffered saline containing $2 \%$ bovine serum albumin (BSA) and resuspended to $5 \times 10^{6}$ cells $\mathrm{mL}^{-1}$. Cells were incubated with 2.3-230 nM iodinated mAb, in the presence and absence of $1.33 \mu \mathrm{M}$ cold antibody, for $1 \mathrm{~h}$ at $4^{\circ} \mathrm{C}$. TS2/18 and $35.1 \mathrm{mAbs}$ displayed saturation binding to cells. Because this binding could have represented bivalent or monovalent binding to cell surface CD2 molecules, mAb molecular weights of 75,000 and 150,000 were used to calculate the limits of a range of specific activities. Bound and free counts were separated by centrifugation at $4000 \times g$ for 3 min through an oil cushion (1.5 parts dibutylphthalate to 1 part dioctylphthalate (41)) in prechilled microsediment tubes. The cell pellet and supernatant were separated and counted. Scatchard analysis was used to calculate the average number of CD2 sites per cell, as described (24).

Preparation of Planar Bilayers Reconstituted with FITC-CD58. GPI-CD58 was conjugated to FITC as previously described (25). To remove additional free FITC, the FITC-CD58 conjugate was subjected to ultrafiltration using a 30,000 MW cut off membrane (Centricon, Amicon) immediately after elution from the affinity column.

Unilamellar liposomes were prepared from egg phosphatidylcholine (PC) (Avanti Polar Lipids). Liposomes with or without reconstituted CD58 were prepared by OG dialysis $(22,42-44)$, stored under an argon atmosphere at $4{ }^{\circ} \mathrm{C}$, and used to prepare glass-supported planar bilayers (22, 42-44). Briefly, $10 \mu \mathrm{L}$ of liposome suspension was deposited at the bottom of a plastic Petri dish $(50 \mathrm{~mm} \times 9 \mathrm{~mm})$, and a cleaned glass coverslip was placed on the droplet to fuse the liposomes at the glass surface. The coverslip was incubated for $30 \mathrm{~min}$ at RT and then gently washed with binding buffer containing $25 \mathrm{mM} \mathrm{HEPES} \mathrm{(} \mathrm{pH}$ 7.4), $147 \mathrm{mM} \mathrm{NaCl}, 5 \mathrm{mM} \mathrm{KCl}, 0.8 \mathrm{mM} \mathrm{MgCl}, 1.8 \mathrm{mM} \mathrm{CaCl}, 5 \mathrm{mM}$ D-glucose, and 1\% BSA (Calbiochem). The coverslip was rinsed 3-5 times with binding buffer and then immersed in a tank containing $1 \mathrm{~L}$ of binding buffer. A stainless steel slide with a 15-mm-diam hole at the center was coated on both sides with vacuum grease and a clean blank coverslip was sealed to one side of the slide. The slide was immersed in the tank, and the bilayer-coated coverslip was carefully mounted to the other side of the slide, with the bilayer facing the inside of the hole. The slide assembly was removed from the tank and rinsed with distilled water. The blank coverslip was carefully removed to open the chamber, and cells or reagents were added. The coverslip was then replaced to seal the chamber. Planar bilayers were not exposed to open air during the procedure. The presence of GPICD58 molecules in the bilayer was confirmed by the specific binding of fluorescent antibodies. We observed that all of the reconstituted FITC-CD58 molecules were oriented away from the glass surface (23).

Imaging and Analysis. Two-dimensional $108 \mu \mathrm{m} \times 108 \mu \mathrm{m}$ fluorescence images of FITC-CD58 redistribution in egg PC bilayers were acquired using a laser-scanning confocal fluorescence microscope (Meridian ACAS 570) with a pinhole diameter of $225 \mu \mathrm{m}$ and a step size of $0.6 \mu \mathrm{m}$. The laser excitation wavelength was $514 \mathrm{~nm}$. The focus was adjusted to the plane of the bilayer as determined by the maximum fluorescence intensity. A quantitative measure of cellular autofluorescence and background fluorescence was obtained from cells in contact with bilayers containing unlabeled CD58. Cells did not bind to CD58containing bilayers in the presence of the adhesion-blocking CD2 mAb TS2/18 (data not shown). The contact area of a cell with a bilayer $\left(S_{b}\right)$ was defined as the area of the accumulated fluorescence under the cell; the fluorescence intensity threshold for each image was set equal to the sum of cellular autofluorescence/background fluorescence and the free FITC-CD58 fluorescence intensity $(F)$, where $F$ was measured from a cell-free area of the bilayer (vide infra). Contact area determinations using this method were in generally good agreement with those determined using interference reflection microscopy (25). The fluorescence intensity was converted to CD58 surface density using a standard curve of fluorescence intensity vs FITC-CD58 density, as determined by the binding of ${ }^{125}$-labeled anti-CD58 mAb TS2/9. The free (or unbound) CD58 density $(F)$ in the bilayer was determined by the fluorescence intensity in a cell-free area after subtraction of the background fluorescence of a bare glass coverslip. Cellular autofluorescence/background fluorescence and free FITC-CD58 fluorescence $(F)$ were then subtracted from the fluorescence intensity in the cell-bilayer contact area to yield the surface density of bound CD58 (B).

FPR. Fluorescence photobleaching recovery (FPR) was used to measure the fractional mobility and lateral diffusion coefficient of FITC-CD58 in the planar bilayer membrane and of FITC-mAblabeled CD2 in the T cell membrane. Experiments were performed using a confocal laser-scanning microscope (Meridian ACAS 570) as described $(22,25)$. FITC-labeled anti-CD2 mAbs TS2/18 (adhesion-blocking) and CD2.1 (non-adhesion-blocking) were used to label CD2 molecules on Jurkat cells. FPR measurements of CD2 mobility were performed at either the cell bilayer contact area or the upper surface of the cell. For the latter experiments, the focal plane of the microscope was first oriented at the cell-bilayer contact plane and then moved up by $10 \mu \mathrm{m}$ from the plane of the bilayer to a region at the upper surface of the cell. (N.B., the average diameter of Jurkat cells was $11.1 \mu \mathrm{m}$.) All measurements were carried out at RT.

2D Affinity Analysis. Scatchard analysis cannot be used to measure the affinity of receptors at a contact area in which the 
receptors are accumulating over time. Instead, we have derived a new analysis that takes account of this dynamic condition, and we have successfully used the analysis to measure the $2 \mathrm{D} K_{\mathrm{d}}$ of the $\mathrm{CD} 2-\mathrm{CD} 58$ interaction in resting Jurkat T cells $(23,24)$. Briefly, the analysis uses quantitative fluorescence imaging to determine both the number of receptors in the contact area and the size of the contact area. FPR is then used to determine the fraction of laterally mobile receptors. The formation of $\mathrm{CD} 2-$ CD58 complexes in the contact area is described by the general Zhu-Golan equation:

$$
\frac{B}{F}=\frac{N_{t} \times f}{K_{d} \times S_{\text {cell }}}-\frac{B \times p}{K_{d}}
$$

where $B$ is the density of bound $C D 58$ in the contact area, $F$ is the density of free CD58 in the bilayer, $N_{\mathrm{t}}$ is the total number of CD2 binding sites on the cell, $f$ is the percentage of laterally mobile CD2 on the cell, $K_{\mathrm{d}}$ is the $2 \mathrm{D}$ dissociation constant, $S_{\text {cell }}$ is the total surface area of the cell, and $p$ is the ratio of the contact area $\left(S_{b}\right)$ to $S_{\text {cell }}\left(i . e ., p=S_{b} / S_{\text {cell }}\right)$. $\left(N_{t} \times f\right)$ represents the number of available binding sites, since immobile receptors are unable to migrate into the contact area. According to eq 1 , we can plot the data derived from fluorescence imaging and FPR experiments according to the Zhu-Golan form:

$$
\frac{B}{F} v s B \times p
$$

This plot generates a line with a negative reciprocal slope equal to the $2 \mathrm{D} K_{\mathrm{d}}$, and an $X$-intercept $(X)$ that can be used to determine $N_{\mathrm{t}}$ from the following relationship:

$$
N_{t}=\frac{\left(X \times S_{\text {cell }}\right)}{f}
$$

SPT. Jurkat cells were used for all SPT experiments. Cells were cultured as described above and labeled with beads as described elsewhere $(27,28)$. Briefly, cells were harvested from cultures in exponential growth and washed three times with Hank's balanced salt solution (HBSS) supplemented with $1 \%(\mathrm{~m} / \mathrm{v}) \mathrm{BSA}$ (HBSSB). The cells were then resuspended in HBSSB containing DMSO $(0.1 \% \mathrm{v} / \mathrm{v})$, DMSO with PMA $(150 \mathrm{nM})$, or DMSO with cytochalasin D (cytoD) $\left(5 \mu \mathrm{g} \mathrm{mL}^{-1}\right)$. Aliquots of the cell suspension $(0.25 \mathrm{~mL}$ ) were incubated for $30 \mathrm{~min}$ at $37^{\circ} \mathrm{C}$ and then labeled with polystyrene microspheres (vide infra) for another $15 \mathrm{~min}$ at $37^{\circ} \mathrm{C}$. The samples were then diluted to $1 \mathrm{~mL}$ with HBSSB and transferred to a 24-well plate containing $12 \mathrm{~mm}$ circular coverslips treated with Cell-Tak (BD Pharmingen). The plate was centrifuged at $\sim 500 \mathrm{rpm}$ for $7 \mathrm{~min}$, and then the wells were carefully washed seven times with fresh HBSSB $(1 \mathrm{~mL})$. One of the coverslips was then transferred to a microslide, seated using a thin circle of vacuum grease, and sealed with Cytoseal 60 (Richard-Allan Scientific). Samples were observed at $37^{\circ} \mathrm{C}$ within 90 min of sealing.

One-micrometer polystyrene microspheres were obtained from Polysciences $(2.6 \% \mathrm{~m} / \mathrm{v})$. The beads were diluted to a stock solution of $1.3 \%(\mathrm{~m} / \mathrm{v})$ in deionized water with $0.1 \% \mathrm{NaN}_{3}$ and sonicated for 15 min before each use. Beads $(10 \mu \mathrm{L}$ of stock solution) were labeled by incubation with monoclonal $\mathrm{F}(\mathrm{ab})^{\prime}$ fragments or purified GPI-CD58 $(0.1-10 \mu \mathrm{g})$ for $1 \mathrm{~h}$ in $0.2 \mathrm{~mL}$ of borate buffer (100 mM borate, $1 \mathrm{mM}$ EDTA, $0.1 \% \mathrm{NaN}_{3}, \mathrm{pH}=$ 8.5) at a final bead concentration of $0.05 \%(\mathrm{~m} / \mathrm{v})$. In all cases, we used the minimum amount of protein required to achieve selective binding of beads to cells. Control beads were labeled with polyclonal $\mathrm{F}(\mathrm{ab})^{\prime}$ or BSA under identical conditions. After adsorption of the protein to the beads, the samples were diluted to $1 \mathrm{~mL}$ with blocking buffer (10 mM HEPES, $140 \mathrm{mM} \mathrm{NaCl}, 1 \mathrm{mM}$
EDTA, $2 \%$ dextran, $1 \% \mathrm{BSA}, 0.1 \% \mathrm{NaN}_{3}, 0.1 \mu \mathrm{g} \mathrm{mL} \mathrm{L}^{-1}$ poly(ethylene glycol) compound, pH 7.4) and incubated for $1 \mathrm{~h}$. The samples were then sonicated for $15 \mathrm{~min}$ and centrifuged at 5000 $\mathrm{rpm}$ for $7 \mathrm{~min}$. The supernatant was aspirated to $0.1 \mathrm{~mL}$ final volume, resuspended, and sonicated for $15 \mathrm{~min}$ immediately before use. All labeled bead samples were used within $48 \mathrm{~h}$ of preparation. Selectivity of binding was confirmed at the beginning of each experiment by manually counting the number of positively labeled cells in $\sim 20$ random fields. Samples were used for tracking experiments if the selectivity of bead binding was $>4$-fold greater than the negative control. Bead binding was typically $0.4 \%$ of cells for control beads, $2.8 \%$ (7-fold selectivity) for TS2/18-labeled beads, and 2\% (5-fold selectivity) for CD58labeled beads.

Cells were observed on a Nikon TE2000-E microscope equipped with DIC optics using a $60 \times$ oil objective with an oil condenser $(\mathrm{NA}=1.4)(45)$. Images were captured at $1000 \mathrm{FPS}$ using a Fastcam Super 10K camera (Photron). Video data were processed using Metamorph (Universal Imaging) and converted to trajectories. Trajectory data were analyzed using mean square displacement analysis (28) implemented in a custom program written in Matlab (Mathworks) as described (27).

Acknowledgments: C.W.C. was funded by NIH NRSA Postdoctoral Fellowship F32 GM067292 and NIH Training Grant T32 HL07623. D.E.G. acknowledges funding from NIH Grants HL32854 and HL70819, and M.L.D. acknowledges funding from NIH Grant Al43542.

Supporting Information Available: This material is available free of charge via the Internet.

\section{REFERENCES}

1. Bachmann, M. F., Barner, M., and Kopf, M. (1999) CD2 sets quantitative thresholds in T cell activation, J. Exp. Med. 190, 1383-1391.

2. Shaw, S., Luce, G. E. G., Quinones, R., Gress, R. E., Springer, T. A., and Sanders, M. E. (1986) Two antigen-independent adhesion pathways used by human cytotoxic T-cell clones, Nature 323, 262-264.

3. Dustin, M. L., Sanders, M. E., Shaw, S., and Springer, T. A. (1987) Purified lymphocyte function-associated antigen-3 binds to CD2 and mediates lymphocyte-T adhesion, J. Exp. Med. 165, 677-692.

4. Moingeon, P., Chang, H. C., Wallner, B. P., Stebbins, C., Frey, A. Z., and Reinherz, E. L. (1989) CD2-mediated adhesion facilitates lymphocyte-T antigen recognition function, Nature 339, 312-314.

5. Tibaldi, E. V., Salgia, R., and Reinherz, E. L. (2002) CD2 molecules redistribute to the uropod during T cell scanning: Implications for cellular activation and immune surveillance, Proc. Natl. Acad. Sci. U.S.A. 99, 7582-7587.

6. Dustin, M. L., Olszowy, M. W., Holdorf, A. D., Li, J., Bromley, S., Desai, N., Widder, P., Rosenberger, F., van der Merwe, P. A., Allen, P. M., and Shaw, A. S. (1998) A novel adaptor protein orchestrates receptor patterning and cytoskeletal polarity in T-cell contacts, Cell 94, 667-677.

7. Wild, M. K., Cambiaggi, A., Brown, M. H., Davies, E. A., Ohno, H., Saito, T., and van der Merwe, P. A. (1999) Dependence of T cell antigen recognition on the dimensions of an accessory receptorligand complex, J. Exp. Med. 190, 31-41.

8. Bromley, S. K., Burack, W. R., Johnson, K. G., Somersalo, K., Sims, T. N., Sumen, C., Davis, M. M., Shaw, A. S., Allen, P. M., and Dustin, M. L. (2001) The immunological synapse, Annu. Rev. Immunol. 19, 375-396.

9. Doyle, C., and Strominger, J. L. (1987) Interaction between CD4 and class-II MHC molecules mediates cell-adhesion, Nature 330, 256-259.

10. Luescher, I. F., Vivier, E., Layer, A., Mahiou, J., Godeau, F., Malissen, B., and Romero, P. (1995) CD8 modulation of T-cell antigen receptor-ligand interactions on living cytotoxic T-lymphocytes, Nature $373,353-356$. 
11. Matsui, K., Boniface, J. J., Reay, P. A., Schild, H., Destgroth, B. F., and Davis, M. M. (1991) Low affinity interaction of peptide-MHC complexes with T-cell receptors, Science 254, 1788-1791.

12. O’Rourke, A. M., Apgar, J. R., Kane, K. P., Martz, E., and Mescher, M. F. (1991) Cytoskeletal function in CD8-cell and T-cell receptormediated interaction of cytotoxic lymphocytes-T with class-I protein, J. Exp. Med. 173, 241-249.

13. Weber, S., Traunecker, A., Oliveri, F., Gerhard, W., and Karjalainen, K. (1992) Specific low-affinity recognition of major histocompatibility complex plus peptide by soluble T-cell receptor, Nature 356, 793-796.

14. Irvine, D. J., Purbhoo, M. A., Krogsgaard, M., and Davis, M. M. (2002) Direct observation of ligand recognition by T cells, Nature 419 , 845-849.

15. Hahn, W. C., Burakoff, S. J., and Bierer, B. E. (1993) Signal transduction pathways involved in T-cell receptor-induced regulation of CD2 avidity for CD58, J. Immunol. 150, 2607-2619.

16. Davis, S. J., Ikemizu, S., Wild, M. K., and van der Merwe, P. A. (1998) $\mathrm{CD} 2$ and the nature of protein interactions mediating cell-cell recognition, Immunol. Rev. 163, 217-236.

17. Dustin, M. L., Olive, D., and Springer, T. A. (1989) Correlation of CD2 binding and functional-properties of multimeric and monomeric lymphocyte function-associated antigen-3, J. Exp. Med. 169, 503-517.

18. van der Merwe, P. A., Barclay, A. N., Mason, D. W., Davies, E. A., Morgan, B. P., Tone, M., Krishnam, A. K. C., Ianelli, C., and Davis, S. J. (1994) Human cell-adhesion molecule CD2 binds CD58 (LFA-3) with a very-low affinity and an extremely fast dissociation rate but does not bind CD48 or CD59, Biochemistry 33, 10149-10160.

19. Bell, G. I., Dembo, M., and Bongrand, P. (1984) Cell-adhesion - competition between nonspecific repulsion and specific bonding, Biophys. J. 45, 1051-1064.

20. Bell, G. I. (1978) Models for the specific adhesion of cells to cells, Science 200, 618-627.

21. McConnell, H. M., Watts, T. H., Weis, R. M., and Brian, A. A. (1986) Supported planar membranes in studies of cell-cell recognition in the immune system, Biochim. Biophys. Acta 864, 95-106.

22. Dustin, M. L., Ferguson, L. M., Chan, P. Y., Springer, T. A., and Golan, D. E. (1996) Visualization of CD2 interaction with LFA-3 and determination of the two-dimensional dissociation constant for adhesion receptors in a contact area, J. Cell Biol. 132, 465-474.

23. Zhu, D. M., Dustin, M.L., Cairo, C.W., and Golan, D. E. (2006) Analysis of two-dimensional dissociation constant of laterally mobile cell adhesion molecules, Biophys. J., in press.

24. Dustin, M. L. (1997) Adhesive bond dynamics in contacts between T lymphocytes and glass-supported planar bilayers reconstituted with the immunoglobulin-related adhesion molecule CD58, J. Biol. Chem. 272, 15782-15788.

25. Dustin, M. L., Golan, D. E., Zhu, D. M., Miller, J. M., Meier, W., Davies, E. A., and van der Merwe, P. A. (1997) Low affinity interaction of human or rat $\mathrm{T}$ cell adhesion molecule CD2 with its ligand aligns adhering membranes to achieve high physiological affinity, J. Biol. Chem. 272, 30889-30898.

26. Liu, S. J., Hahn, W. C., Bierer, B. E., and Golan, D. E. (1995) Intracellular mediators regulate CD2 lateral diffusion and cytoplasmic Ca2+ mobilization upon CD2-mediated T-cell activation, Biophys. J. 68, 459-470.

27. Cairo, C. W., Mirchev, R., and Golan, D. E. (2006) Cytoskeletal regulation couples LFA-1 conformational changes to receptor lateral mobility and clustering, Immunity 25, 297-308.

28. Saxton, M. J., and Jacobson, K. (1997) Single-particle tracking: Applications to membrane dynamics, Annu. Rev. Biophys. Biomol. Struct. $26,373-399$.
29. Gunzer, M., Schafer, A., Borgmann, S., Grabbe, S., Zanker, K. S. Brocker, E. B., Kampgen, E., and Friedl, P. (2000) Antigen presentation in extracellular matrix: Interactions of $T$ cells with dendritic cells are dynamic, short lived, and sequential, Immunity 13, 323-332.

30. Mege, J. L., Capo, C., Benoliel, A. M., Foa, C., Galindo, R., and Bongrand, P. (1986) Quantification of cell-surface roughness - A method for studying cell mechanical and adhesive properties, J. Theor. Biol. $119,147-160$

31. Bockenstedt, L. K., Goldsmith, M. A., Dustin, M., Olive, D., Springer, T. A., and Weiss, A. (1988) The CD2 ligand LFA-3 activates T-cells but depends on the expression and function of the antigen receptor, J. Immunol. 141, 1904-1911.

32. Li, J., Smolyar, A., SunderPlassmann, R., and Reinherz, E. L. (1996) Ligand-induced conformational change within the CD2 ectodomain accompanies receptor clustering: Implication for molecular lattice formation, J. Mol. Biol. 263, 209-226.

33. Kitao, A., and Wagner, C. (2000) A space-time structure determination of human CD2 reveals the CD58-binding mode, Proc. Natl. Acad. Sci. U.S.A. 97, 2064-2068.

34. Wang, J., Smolyar, A., Tan, K. M., Liu, J., Kim, M. Y., Sun, Z. J., Wagner, G., and Reinherz, E. L. (1999) Structure of a heterophilic adhesion complex between the human CD2 and CD58 (LFA-3) counterreceptors, Cell 97, 791-803.

35. Wyss, D. F., Dayie, K. T., and Wagner, G. (1997) The counterreceptor binding site of human CD2 exhibits an extended surface patch with multiple conformations fluctuating with millisecond to microsecond motions, Protein Sci. 6, 534-542.

36. Shaw, A. S., and Dustin, M. L. (1997) Making the T cell receptor go the distance: A topological view of T cell activation, Immunity 6 , 361-369.

37. Alberola-Ila, J., Places, L., Delacalle, O., Romero, M., Yague, J., Gallart, T., Vives, J., and Lozano, F. (1991) Stimulation through the TCR/ CD3 complex up-regulates the CD2 surface expression on human lymphocytes-T, J. Immunol. 146, 1085-1092.

38. Thatte, H. S., Bridges, K. R., and Golan, D. E. (1994) Microtubule inhibitors differentially affect translational movement, cell-surface expression, and endocytosis of transferrin receptors in K562 cells, J. Cell. Physiol. 160, 345-357.

39. Dustin, M. L., Bivona, T. G., and Philips, M. R. (2004) Membranes as messengers in T cell adhesion signaling, Nat. Immunol. 5, 363-372.

40. Hutchings, N. J., Clarkson, N., Chalkley, R., Barclay, A. N., and Brown, M. H. (2003) Linking the T cell surface protein CD2 to the actincapping protein CAPZ via CMS and CIN85, J. Biol. Chem. 278, $22396-22403$.

41. Sayre, P. H., Hussey, R. E., Chang, H. C., Ciardelli, T. L., and Reinherz, E. L. (1989) Structural and binding analysis of a two-domain extracellular CD2 molecule, J. Exp. Med. 169, 995-1009.

42. Brian, A. A., and McConnell, H. M. (1984) Allogeneic stimulation of cyto-toxic T-cells by supported planar membranes, Proc. Natl. Acad. Sci. U.S.A. 81, 6159-6163.

43. Chan, P. Y., Lawrence, M. B., Dustin, M. L., Ferguson, L. M., Golan, D. E., and Springer, T. A. (1991) Influence of receptor lateral mobility on adhesion strengthening between membranes containing LFA-3 and CD2, J. Cell Biol. 115, 245-255.

44. Mimms, L. T., Zampighi, G., Nozaki, Y., Tanford, C., and Reynolds, J. A. (1981) Phospholipid vesicle formation and transmembrane protein incorporation using octyl glucoside, Biochemistry 20, 833-840.

45. Mirchev, R., and Golan, D. E. (2001) Single-particle tracking and laser optical tweezers studies of the dynamics of individual protein molecules in membranes of intact human and mouse red cells, Blood Cells Mol. Dis. 27, 143-147. 